\title{
Trends in neurosurgical care in Western Uganda: an interrupted time series analysis
}

\author{
Jihad Abdelgadir, MD, MSc, ${ }^{1,2}$ Cyrus Elahi, ${ }^{2,3}$ Jacquelyn Corley, MD,,2 Kevin C. Wall, ${ }^{4}$ \\ Josephine N. Najjuma, BNS, ${ }^{5}$ Alex Muhindo, MD, ${ }^{6}$ Joao Ricardo Nickenig Vissoci, PhD, ${ }^{2,3}$ \\ Michael M. Haglund, MD, PhD, MACM, ${ }^{1-3}$ and David Kitya, MD ${ }^{5}$
}

\begin{abstract}
1Department of Neurosurgery, Duke University Medical Center; ${ }^{2}$ Duke Division of Global Neurosurgery and Neurology; ${ }^{3}$ Duke University Global Health Institute; ${ }^{4}$ Duke University School of Medicine, Durham, North Carolina; ${ }^{5}$ Department of Neurosurgery, Mbarara Regional Referral Hospital, Mbarara; and ${ }^{6}$ Department of Neurosurgery, Mulago National Referral Hospital, Kampala, Uganda
\end{abstract}

OBJECTIVE In addition to the rising burden of surgical disease globally, infrastructure and human resources for health remain a great challenge for low- and middle-income countries, especially in Uganda. In this study, the authors aim to explore the trends of neurosurgical care at a regional referral hospital in Uganda and assess the long-term impact of the institutional collaboration between Mulago National Referral Hospital and Duke University.

METHODS An interrupted time series is a quasi-experimental design used to evaluate the effects of an intervention on longitudinal data. The authors applied this design to evaluate the trends in monthly mortality rates for neurosurgery patients at Mbarara Regional Referral Hospital (MRRH) from March 2013 to October 2015. They used segmented regression and autoregressive integrated moving average models for the analysis.

RESULTS Over the study timeframe, MRRH experienced significant increases in referrals received (from 117 in 2013 to 211 in 2015), neurosurgery patients treated (from 337 in 2013 to 625 in 2015), and operations performed (from 61 in 2013 to 173 in 2015). Despite increasing patient volumes, the hospital achieved a significant reduction in hospital mortality during 2015 compared to prior years ( $p$ value $=0.0039$ ).

CONCLUSIONS This interrupted time series analysis study showed improving trends of neurosurgical care in Western Uganda. There is a steady increase in volume accompanied by a sharp decrease in mortality through the years. Multiple factors are implicated in the significant increase in volume and decrease in mortality, including the addition of a part-time neurosurgeon, improvement in infrastructure, and increased experience. Further in-depth prospective studies exploring seasonality and long-term outcomes are warranted.

https://thejns.org/doi/abs/10.3171/2018.7.FOCUS18270

KEYWORDS global neurosurgery; global surgery; LMICs; low- and middle-income countries

$\mathrm{T}$ HE current state of Sub-Saharan African neurosurgery is burdened with a poverty of resources and in need of further research. Indeed, the commonly cited statistic that there is 1 neurosurgeon in Sub-Saharan Africa (SSA), excluding South Africa, for every 6,368,000 inhabitants is taken from a report published in $1998 .{ }^{5}$ This is in contrast to the density of neurosurgeons per capita in the United States, which was 1 per 55,000 in 2000. ${ }^{18}$ The report used the approximated population of 515 million for this group of countries, and the aforementioned ratio of 1/6,368,000 was calculated using the known number of neurosurgeons to be 81 . As of 2016, the population of these nations has doubled, and is now 1.033 billion (https://data. worldbank.org/indicator/SP.POP.TOTL). For that ratio to have held constant, the number of neurosurgeons practicing in this region would need to have increased with the population to be at 148 in 2015.

To put the current ratio of Sub-Saharan African neurosurgeons per capita in perspective, it is 60-fold less than the 1:100,000 ratio seen in the United States. ${ }^{19}$ In an at-

ABBREVIATIONS ARIMA = autoregressive integrated moving average; DGH = Duke Global Health Institute; DUMC = Duke University Medical Center; GCS = Glasgow Coma Scale; ITS = interrupted time series; MNRH = Mulago National Referral Hospital; MRRH = Mbarara Regional Referral Hospital; SSA = Sub-Saharan Africa. SUBMITTED May 31, 2018. ACCEPTED July 23, 2018. INCLUDE WHEN CITING DOI: 10.3171/2018.7.FOCUS18270. 
tempt to increase the number of neurosurgeons in these countries, numerous residency, or other, less-formal training, programs have been created. Hospitals in countries such as Tanzania ${ }^{25}$ and $\mathrm{Uganda}^{8}$ serve as examples of such programs, but there remains a dearth of literature on the longitudinal efficacy of these programs and the presence of others. One recently published article on this topic demonstrates that, in the existing neurosurgery departments in SSA, physicians feel insufficiently prepared; $35 \%$ of survey responders report that they had inadequate training, $28 \%$ report no formal training program, and only $37 \%$ report adequate training. ${ }^{20}$

The majority of the literature surrounding new or expanding neurosurgery departments in SSA revolves around the previously mentioned topics of supply and demand, training deficiencies, and barriers. It is equally important to study the effects that such new departments have, as they are often the product of international collaborations and have their own shares of failures and pitfalls. ${ }^{12}$ In 2007 , the Mulago National Referral Hospital (MNRH) of Kampala, Uganda, Duke University Medical Center (DUMC), and Duke Global Health Institute (DGHI) formed a neurosurgical twinning program whose effects have been empirically studied. ${ }^{9}$ Notably, this partnership increased total neurosurgical cases, as well as their complexity and efficiency. Similarly, in 2012, a neurosurgery department with one neurosurgeon was founded at Mbarara Regional Referral Hospital (MRRH) to address local surgical need in this region. In January of 2015, one of the neurosurgeon graduates of the MNRH-Duke residency program joined MRRH on an intermittent basis. In this paper, we assess the ongoing progress and expansion of the neurosurgery department at MRRH over time as its caseload increases and the infrastructure and human resources improve.

\section{Methods}

\section{Sample and Setting}

This study is a time series analysis of retrospectively collected data. Patients admitted to MRRH with a neurosurgical condition between 2013 and 2015 were included in the study. MRRH has a neurosurgery department that was established in 2012, and it employs one full-time neurosurgeon. The department has an annual caseload of approximately 600-700, and 200-250 surgeries are performed per year. Further details about acquisition of data have been described elsewhere. ${ }^{2}$ Approval for the study was obtained from Makerere University School of Medicine Research and Ethics Committee, Mbarara University of Science and Technology Research Ethics Committee, and Duke University Health System Institutional Review Board.

\section{Study Design}

A time series analysis is a method for analyzing longitudinal data. ${ }^{21}$ An interrupted time series (ITS) includes an intervention, allowing the investigator to compare pre- and postintervention trends. The intervention in this study was implemented in January 2015. The preintervention period included 22 months (March 2013 to December 2014) and the postimplementation period spanned 10 months (January 2015 to October 2015). The intervention captures a capacity increase at MRRH. Also, a neurosurgery resident, graduate from the MNRH-DUMC-DGHI twinning residency program, began intermittent work at MRRH as a part-time neurosurgeon in January 2015. The new neurosurgeon was only responsible for performing surgeries once per week and was not involved in pre- or postoperative care. Prior to January 2015, MRRH had only one neurosurgeon.

\section{Statistical Analysis}

An ITS was performed using autoregressive integrated moving average (ARIMA) and segmented regression models to analyze the impact of our intervention on the overall mortality rate and the mortality rate for neurosurgery patients at MRRH. For the ARIMA model, the augmented Dickey-Fuller test was performed to assess for stationary data. Two differences were needed to achieve stationary data ( $\mathrm{p}<0.01)$. Next, Akaike's information criterion, bias-corrected Akaike's information criterion, and Bayesian information criterion were compared to identify the ARIMA model with the best fit. We used the ARIMA model to calculate the monthly decrease in mortality rate after the intervention. The segmented regression model allowed us to compare the monthly mortality rate before and after the intervention. By specifying a linear regression model for the pre- and postimplementation segments, we can calculate the monthly mortality trend before the intervention, the change in the level, and the trend following the intervention. Data management and statistical analyses were performed using $\mathrm{R}$ version 3.4.1.

\section{Results}

The number of patients admitted to the inpatient neurosurgery ward was 337 in 2013, 542 in 2014, and 625 in 2015 (Table 1). The number of neurosurgeries performed also increased each year with 61 procedures (6.1 per month) performed in 2013, 137 procedures (11.4 per month) performed in 2014, and 173 procedures (17.3 per month) performed in 2015. The largest changes in procedures performed between 2015 and 2014 were spinal surgeries (28 vs 9), craniotomies (73 vs 63), and ventriculostomies (7 vs 1). The ratio of males to females was about $3: 1$ for the duration of the study. Similarly, the ratio of adult to pediatric patients was also 3:1. The average age for adult patients was 36.6 years, and the average age for pediatric patients was 7.2 years. There was no significant difference in the patient demographic characteristics between the different years of the study.

The length of stay decreased each year of the study, from 10.67 days in 2013 , to 8.83 days in 2014, and to 8.45 days in 2015. The admission Glasgow Coma Scale (GCS) score did change from 2013 to 2014. In 2013, the percentage of patients with an admission GCS score of 13-15, $9-12$, and $3-8$ was $47 \%, 26 \%$, and $16 \%$, respectively. In 2014 , the percentage of patients with a GCS score of 13-15 increased to $57 \%$, while the percentage with a GCS score of $9-12$ decreased from $26 \%$ to $21 \%$. The percentage of patients with a GCS score of 3-8 also decreased between 2013 and 2014 from 16\% to 14\%. The change in patient admission GCS score between 2013 and 2014 was significant 
TABLE 1. Variables in the study population across the study period

\begin{tabular}{|c|c|c|c|c|}
\hline Variable & Mar-Dec 2013 & Jan-Dec 2014 & Jan-Oct 2015 & $p$ Value \\
\hline \multicolumn{5}{|l|}{ Clinical volume } \\
\hline Neurosurgery ward patients (avg/mo) & $337(33.7)$ & $542(45.2)$ & $625(62.5)$ & $<0.001$ \\
\hline Ops performed (avg/mo) & $61(6.1)$ & $137(11.4)$ & $173(17.3)$ & $<0.001$ \\
\hline Referrals (avg/mo) & $117(11.7)$ & $161(13.4)$ & $211(21.1)$ & $<0.001$ \\
\hline Median age, yrs (IQR) & $26(17-38)$ & $28(18-38)$ & $27(18.8-40)$ & 0.964 \\
\hline Sex & & & & 0.218 \\
\hline Male & $254(75)$ & $427(79)$ & $466(75)$ & \\
\hline Female & $83(25)$ & $115(21)$ & $159(25)$ & \\
\hline Presenting GCS score & & & & $0.008^{*}$ \\
\hline Mild, 13-15 & $157(47)$ & $311(57)$ & $357(57)$ & \\
\hline Moderate, 9-12 & $89(26)$ & $114(21)$ & $124(20)$ & \\
\hline Severe, 3-8 & $53(16)$ & $77(14)$ & $74(12)$ & \\
\hline \multicolumn{5}{|l|}{ Clinical outcomes } \\
\hline Overall mortality & $57(16.9)$ & $76(14.0)$ & $67(10.7)$ & 0.018 \\
\hline Neurosurgical mortality & $9(14.8)$ & $12(8.8)$ & $8(4.6)$ & 0.038 \\
\hline Median LOS (IQR) & $6(3-11)$ & $5(3-10)$ & $5(3-10)$ & 0.312 \\
\hline \multicolumn{5}{|l|}{ Procedure } \\
\hline Burr hole & $9(15)$ & $43(31)$ & $44(25)$ & $<0.001$ \\
\hline Craniotomy & $30(49)$ & $63(46)$ & $73(42)$ & $<0.001$ \\
\hline Spine surgery & $10(16)$ & $9(7)$ & $28(16)$ & $<0.001$ \\
\hline VP shunt/ventriculostomy & $3(5)$ & $7(5)$ & $10(6)$ & 0.157 \\
\hline Other† & $9(15)$ & $15(11)$ & $23(11)$ & 0.223 \\
\hline
\end{tabular}

$\mathrm{Avg} / \mathrm{mo}=$ average per month; LOS = length of stay; VP = ventriculoperitoneal.

Values are presented as the number (\%) of patients unless otherwise stated.

* The admission GCS score is significantly different between 2013 and $2014(p=0.027)$ but is not significant between 2014 and $2015(p=0.608)$.

$\dagger$ Other includes cranioplasty, surgical toilet, and myelomeningocele repairs.

$(\mathrm{p}=0.027)$. The change in admission GCS score was not significant between 2014 and 2015 ( $\mathrm{p}=0.608)$.

Over the duration of the study, there were significant changes in the types of procedures performed. The procedures increasing in frequency were as follows: burr holes $(\mathrm{p}<0.001)$, craniotomies $(\mathrm{p}<0.001)$, spine surgeries $(\mathrm{p}$ $<0.001)$, ventriculostomies $(\mathrm{p}=0.018)$, and other $(\mathrm{p}=$ 0.223); "other" includes surgical toilet and suturing (STS), myelomeningocele repairs, and cranioplasties. Despite the increasing patient volume and complexity of the procedures performed, the overall neurosurgery ward mortality rate and neurosurgical mortality rate decreased each year. In 2013, the overall mortality rate was $16.9 \%$, and the neurosurgical mortality rate was $14.8 \%$. These numbers decreased to $10.7 \%$ and $4.6 \%$, respectively, in 2015 .

There was no significant increase or decrease in hospital neurosurgery mortality rate prior to the intervention (Fig. 1). Immediately after the intervention, the intercept did increase slightly; however, the finding was not significant (Table 2). The slope for the mortality rate was significantly different from the preimplementation period, indicating a greater decrease in mortality rates after the intervention (Table 2). This decrease in mortality rate occurred despite increasing patient volumes at the hospital (Fig. 2). The ARIMA model suggested about a $3 \%$ decrease in mortality rate each month after the intervention (Table 2). Finally, the seasonality plot (Fig. 3) shows that the hospital mortal- ity rate decreased during the summer and fall of 2013 and 2015 but increased during these seasons in 2014.

\section{Discussion}

In addition to the rising burden of surgical disease, especially traumatic brain injuries, ${ }^{1,22,23}$ the lack of qualified surgical personnel remains one of the greatest challenges in resource-poor settings. ${ }^{3,7,14,17}$ In this interrupted time series analysis study, we look at the trends of neurosurgical care and the effects of adding a part-time neurosurgeon at a regional referral hospital. Previous studies investigating similar partnerships have measured impact through provider skills and confidence levels pre- and postintervention. ${ }^{16}$ We were able to gather data and quantify the impact on a patient outcome level, which showed a significant reduction in the neurosurgical mortality rate at Mbarara Regional Referral Hospital. Our analysis revealed an annual significant increase in the number of neurosurgery patients from 2013 to 2015, as well as a significant increase in the number of surgeries performed each year. This increase in volume was accompanied by a decline in overall and surgical mortality rates. When comparing the mortality rates pre- and postintervention, adding a part-time neurosurgeon had a significant effect. There was a significant decline in mortality rate following the intervention.

These results are echoed by Kushner et al., whose study 


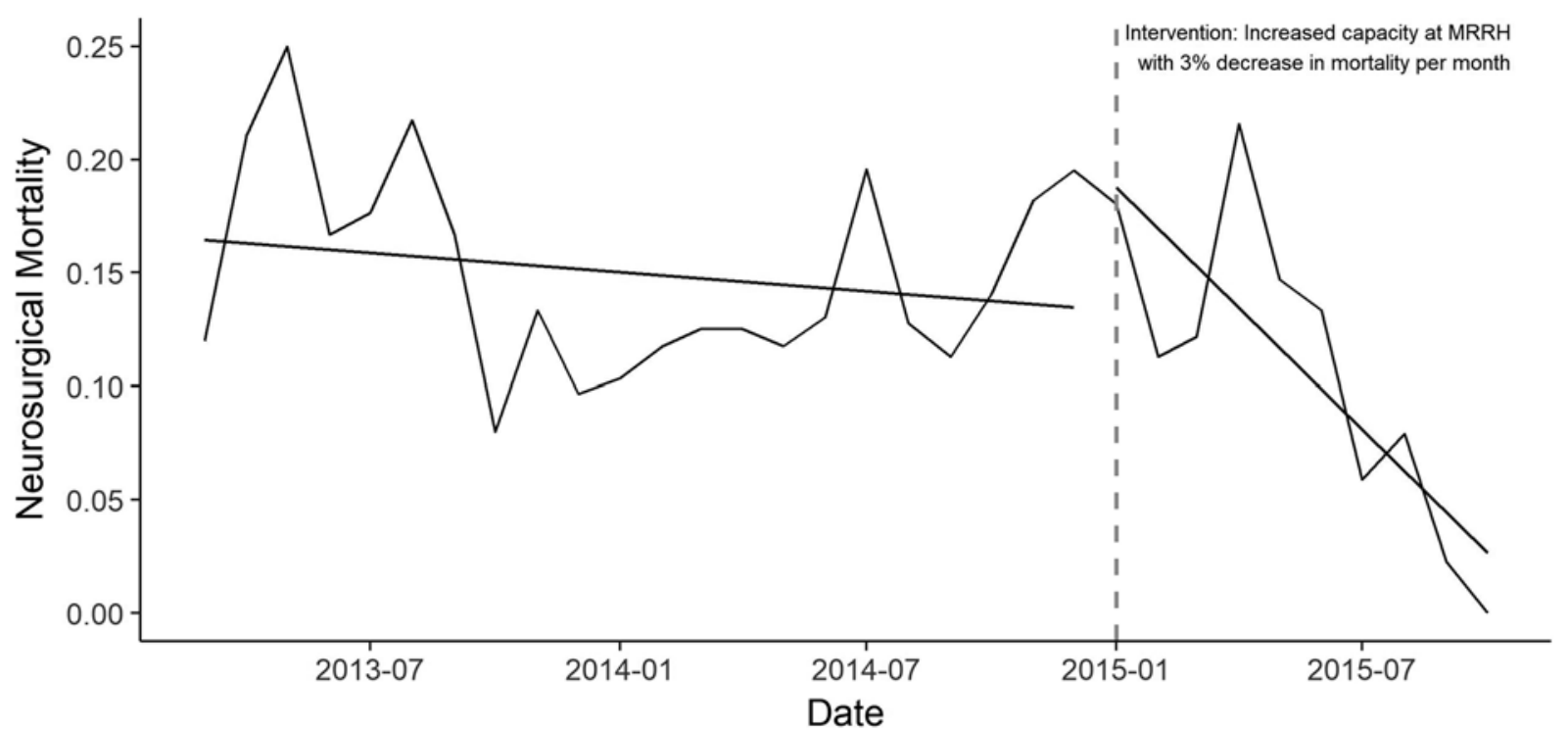

FIG. 1. Monthly MRRH neurosurgery mortality rate from March 2013 to October 2015. The mortality rate includes both surgery and nonsurgery patients. The dashed vertical line represents a part-time neurosurgeon starting once-a-week work at MRRH. The increase in the mortality rate immediately after the intervention is not statistically significant. The change in slope of the mortality rate from before and after the intervention is significant $(p=0.0039)$. Dates: 2013-07 refers to July 2013, 2014-01 refers to January 2014 , and so on.

evaluated a program that provided provision of training, supplies, and salary support to a local hospital in Sierra Leone. These authors demonstrated that access to surgery, as measured by the number of major operations, increased from 460 cases in 2007 to 768 cases in $2009 . .^{13}$ Similarly, a twinning program, implemented in Mulago Hospital in Uganda, boasted an increase in the neurosurgery cases from 125 in 2006-2007 to 392 in 2008-2009. ${ }^{6}$ In our investigation, neurosurgical cases increased from 61 to 173 during the study period. This demonstrates that even a slight increase in human resources - in our case a part-time neurosurgeon who operates once a week-has a tremendous effect on access to surgical care and caseload supported by a health system. Secondary variables may also contribute to this trend in higher case volume. For example, there may be an increase in number of referrals or patients seeking care, as the hospital becomes more recognized for its neurosurgery department.

Our study demonstrates a strong negative correlation

TABLE 2. Estimated coefficients of segmented regression model and ARIMA model for neurosurgical hospital mortality rate

\begin{tabular}{lccc}
\hline \multirow{2}{*}{ Model Parameters } & \multicolumn{3}{c}{ Segmented Regression Model } \\
\cline { 2 - 4 } Estimate $(\mathrm{SE})$ & $\mathrm{t}$ & $\mathrm{p}$ Value \\
\hline Intercept & $0.1660(0.0195)$ & 8.52 & $<0.001$ \\
\hline Preimplementation slope & $-0.0014(0.0015)$ & -0.96 & 0.350 \\
\hline Change in slope & $-0.0165(0.0352)$ & 2.01 & 0.0039 \\
\hline Change in intercept & $0.0708(0.0051)$ & -3.24 & 0.054 \\
\hline \multicolumn{3}{c}{ Intervention } & ARIMA $(1,2,1) \times(1,1,0)_{12}$ Model Output \\
\hline
\end{tabular}

between the annual number of cases and overall mortality. This relationship between hospital volume and outcome has been demonstrated clearly in prior literature., $40,11,15$ The inverse relationship between volume and mortality may be explained by the impact of experience. The clinical staff becomes more experienced and familiar with the cases as the volume increases. This includes nursing staff, ancillary workers, and other providers, who gain more opportunities to develop triaging and critical care skills. This domino effect ultimately improves the quality of care and leads to better outcomes, assuming an appropriate staff-to-patient ratio is maintained. Surgeons themselves become more competent and confident as they become more experienced and are better equipped to manage complications and take on complex cases. It is clear that investing in hospital infrastructure, personnel, and equipment not only increases access to surgical care but also improves outcomes.

The seasonality analysis in this study shows a slight increase in the mortality rate during the summer months. We hypothesize the reason for this to be the lack of rotating residents during these months, leading to decreased workforce and ultimately worse outcomes. Further studies to explore this hypothesis, however, are necessary.

The main limitations of this study result from data quality and collection constraints inherent in paper-based hospital record keeping. Inconsistent recording and storage of data limited the preimplementation period to 22 months. The postimplementation period was only 10 months due to a change in the method used to collect data during the end of 2015. Our postimplementation period of 10 months is 2 months fewer than the general recommendation for using segmented regressions. To appreciate the seasonality of a time series, the literature suggests at least 24 monthly data points. ${ }^{24}$ As a result, our study analysis 


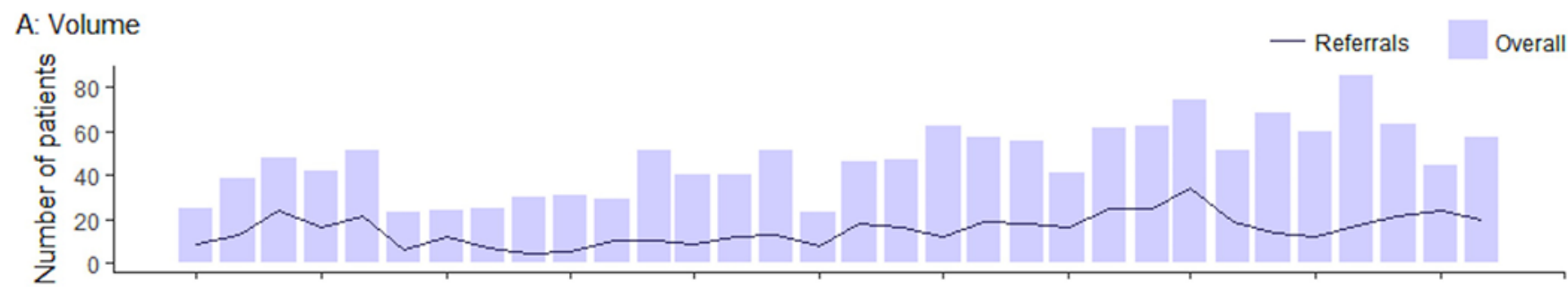

\section{B: Mortality}

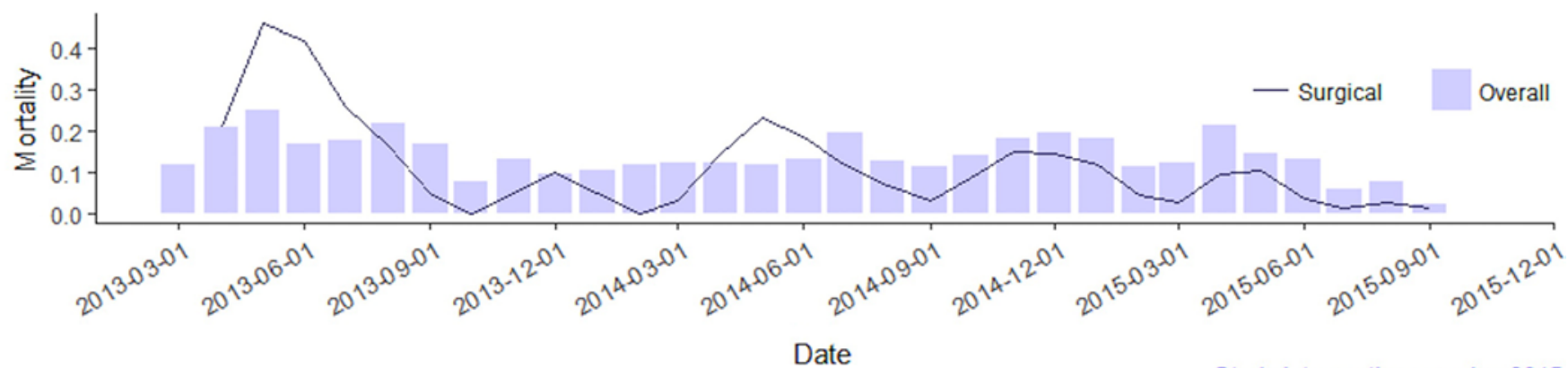

Study intervention was Jan 2015

FIG. 2. Trends in monthly MRRH patient volume and mortality from March 2013 to October 2015. A: The overall (surgery and nonsurgery) volume is shown with the blue bars. The solid blue line represents the number of referrals from other hospitals and clinics to MRRH. B: This graph depicts the MRRH overall (surgery and nonsurgery) neurosurgery mortality rate with the blue bars. The mortality rate for only surgery patients is shown by the solid blue line. Dates: 2013-03-01 refers to March 1, 2013; 2013-06-01 refers to June 1, 2013; and so on.

captures seasonality for the entire study period, but it does not do so for the pre- and postimplementation periods separately. Moreover, we limited our analysis to mortality outcomes, and the data constraints limited our ability to obtain functional short- and long-term outcomes.

Additionally, it should be recognized that these large results and trends are multifactorial and multidisciplinary. Some confounders do exist. For example, during this time, hospital renovations occurred, which may have increased the bed capacity. In 2014, a new CT scanner was installed, and several general surgery resident camps may also have been responsible for volume increase. Overall, the exact causes and effects cannot be elucidated, but rather we can infer the general effect that a new neurosurgeon may have on an entire health system, and likely it will require repetition of results and continued follow-up and analysis.

Notwithstanding these limitations, the implications for this work are broad and far reaching. In a time when there is more enthusiasm for global outreach than ever before, interventions and impact need to be scrutinized for their efficacy and sustainability. History has exposed that even the most well-intentioned efforts have gone awry and had

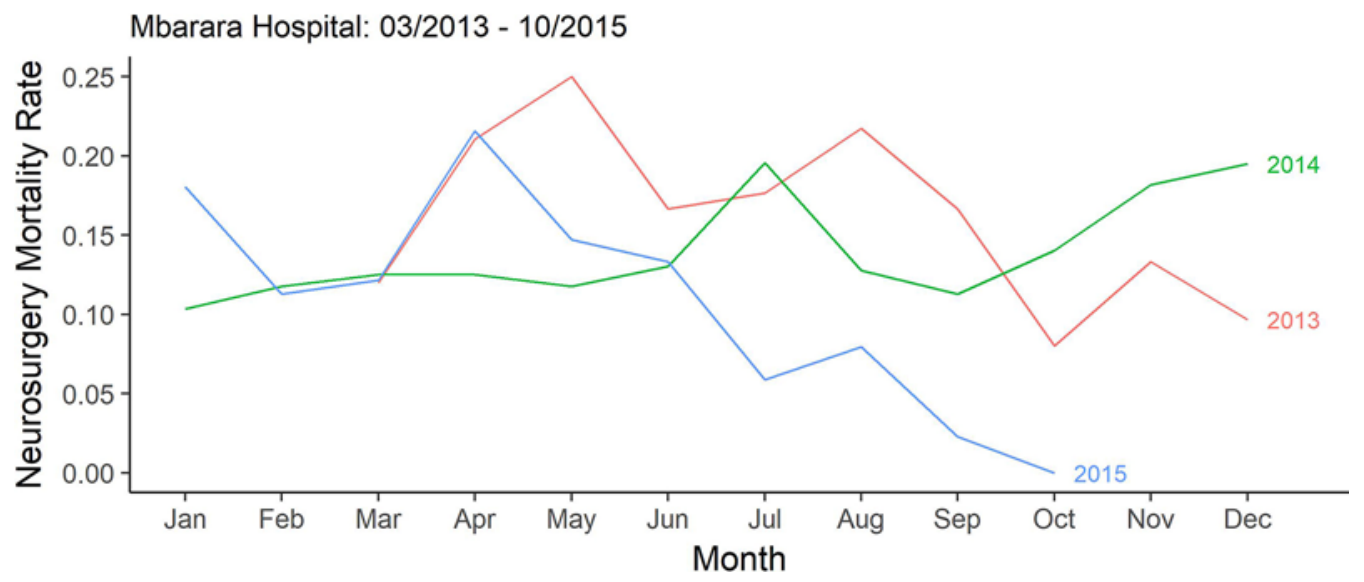

FIG. 3. A seasonality plot for the monthly neurosurgical mortality rate at MRRH for 2013,2014 , and 2015 . The red line plots March-December 2013, the green line plots January-December 2014, and the blue line plots January-October 2015. 
unexpected negative consequences. Sustainable solutions such as this demonstrate that an increase in key healthcare providers can increase overall surgical capacity, which in turn nurtures the growth of an entire health system.

\section{Conclusions}

This study explores the trends of neurosurgical care in Western Uganda and the longitudinal impact of the neurosurgery residency formed through the DUMC-MNRH partnership. The interrupted time series analysis provides empirical evidence supporting investment in hospital infrastructure and human resources. It shows improving trends in neurosurgical care at a regional referral hospital in Western Uganda as the department continues to grow and the system continues to improve. Additional prospective studies assessing factors affecting seasonality of mortality and long-term outcomes are needed.

\section{References}

1. Abdelgadir J, Punchak M, Smith ER, Tarnasky A, Muhindo A, Nickenig Vissoci JR, et al: Pediatric traumatic brain injury at Mbarara Regional Referral Hospital, Uganda. J Clin Neurosci 47:79-83, 2018

2. Abdelgadir J, Smith ER, Punchak M, Vissoci JR, Staton C, Muhindo A, et al: Epidemiology and characteristics of neurosurgical conditions at Mbarara Regional Referral Hospital. World Neurosurg 102:526-532, 2017

3. Choo S, Perry H, Hesse AA, Abantanga F, Sory E, Osen H, et al: Assessment of capacity for surgery, obstetrics and anaesthesia in 17 Ghanaian hospitals using a WHO assessment tool. Trop Med Int Health 15:1109-1115, 2010

4. Courcoulas A, Schuchert M, Gatti G, Luketich J: The relationship of surgeon and hospital volume to outcome after gastric bypass surgery in Pennsylvania: a 3-year summary. Surgery 134:613-623, 2003

5. El Khamlichi A: African neurosurgery. Part II: current state and future prospects. Surg Neurol 49:342-347, 1998

6. Fuller A, Tran T, Muhumuza M, Haglund MM: Building neurosurgical capacity in low and middle income countries. eNeurologicalSci 3:1-6, 2015

7. Groen RS, Kamara TB, Dixon-Cole R, Kwon S, Kingham TP, Kushner AL: A tool and index to assess surgical capacity in low income countries: an initial implementation in Sierra Leone. World J Surg 36:1970-1977, 2012

8. Haglund MM, Kiryabwire J, Parker S, Zomorodi A, MacLeod D, Schroeder R, et al: Surgical capacity building in Uganda through twinning, technology, and training camps. World J Surg 35:1175-1182, 2011

9. Haglund MM, Warf B, Fuller A, Freischlag K, Muhumuza $\mathrm{M}$, Ssenyonjo $\mathrm{H}$, et al: Past, present, and future of neurosurgery in Uganda. Neurosurgery 80:656-661, 2017

10. Hannan EL, O'Donnell JE, Kilburn H Jr, Bernard HR, Yazici A: Investigation of the relationship between volume and mortality for surgical procedures performed in New York State Hospitals. Surv Anesthesiol 34:175, 1990

11. Hollenbeak CS, Rogers AM, Barrus B, Wadiwala I, Cooney RN: Surgical volume impacts bariatric surgery mortality: a case for centers of excellence. Surgery 144:736-743, 2008

12. Ibrahim GM, Bernstein M: Models of neurosurgery international aid and their potential ethical pitfalls. Virtual Mentor 17:49-55, 2015

13. Kushner AL, Kamara TB, Groen RS, Fadlu-Deen BD, Doah KS, Kingham TP: Improving access to surgery in a developing country: experience from a surgical collaboration in Sierra Leone. J Surg Educ 67:270-273, 2010

14. Linden AF, Sekidde FS, Galukande M, Knowlton LM, Chack- ungal S, McQueen KA: Challenges of surgery in developing countries: a survey of surgical and anesthesia capacity in Uganda's public hospitals. World J Surg 36:1056-1065, 2012

15. Luft HS, Bunker JP, Enthoven AC: Should operations be regionalized? The empirical relation between surgical volume and mortality. 1979. Clin Orthop Relat Res 457:3-9, 2007

16. Macpherson L, Collins M: Training responsibly to improve global surgical and anaesthesia capacity through institutional health partnerships: a case study. Trop Doct 47:73-77, 2017

17. Ploss B, Abdelgadir J, Smith ER, Fuller A, Nickenig Vissoci JR, Muhindo A, et al: Pilot use of a novel tool to assess neurosurgical capacity in Uganda. World Neurosurg 108:844849, 849.e1-849.e4, 2017

18. Popp A: Neurosurgical workforce: Examining the physician supply controversy. AANS Bull 9:7-9, 2000

19. Rosman J, Slane S, Dery B, Vogelbaum MA, Cohen-Gadol AA, Couldwell WT: Is there a shortage of neurosurgeons in the United States? Neurosurgery 73:354-366, 2013

20. Sader E, Yee P, Hodaie M: Barriers to neurosurgical training in Sub-Saharan Africa: the need for a phased approach to global surgery efforts to improve neurosurgical care. World Neurosurg 98:397-402, 2017

21. Shumway RH, Stoffer DS: Time Series Analysis and Its Applications: With R Examples, ed 3. New York: Springer, 2011

22. Staton CA, Msilanga D, Kiwango G, Vissoci JR, de Andrade $\mathrm{L}$, Lester R, et al: A prospective registry evaluating the epidemiology and clinical care of traumatic brain injury patients presenting to a regional referral hospital in Moshi, Tanzania: challenges and the way forward. Int J Inj Contr Saf Promot 24:69-77, 2017

23. Tran TM, Fuller AT, Butler EK, Makumbi F, Luboga S, Muhumuza C, et al: Burden of surgical conditions in Uganda: a cross-sectional nationwide household survey. Ann Surg 266:389-399, 2017

24. Wagner AK, Soumerai SB, Zhang F, Ross-Degnan D: Segmented regression analysis of interrupted time series studies in medication use research. J Clin Pharm Ther 27:299-309, 2002

25. Wilson DA, Garrett MP, Wait SD, Kucia EJ, Saguda E, Ngayomela I, et al: Expanding neurosurgical care in Northwest Tanzania: the early experience of an initiative to teach neurosurgery at Bugando Medical Centre. World Neurosurg 77:32-38, 2012

\section{Disclosures}

Dr. Haglund has received clinical or research support from NuVasive and Lifenet. He also has received support of non-studyrelated clinical or research effort from UCB Pharma.

\section{Author Contributions}

Conception and design: Haglund, Abdelgadir, Elahi, Nickenig Vissoci, Kitya. Acquisition of data: Abdelgadir, Najjuma, Muhindo. Analysis and interpretation of data: Abdelgadir, Elahi, Nickenig Vissoci. Drafting the article: Abdelgadir, Elahi, Corley, Wall. Critically revising the article: Haglund, Abdelgadir, Corley, Najjuma, Muhindo, Nickenig Vissoci, Kitya. Reviewed submitted version of manuscript: all authors. Approved the final version of the manuscript on behalf of all authors: Haglund. Statistical analysis: Abdelgadir, Elahi, Nickenig Vissoci. Study supervision: Haglund, Kitya.

\section{Supplemental Information \\ Videos}

Video Abstract. https://vimeo.com/288345869.

\section{Correspondence}

Michael M. Haglund: Duke University, Durham, NC. michael. haglund@duke.edu. 